\title{
Energy-saving potential deriving from the use of a manifold with flow rate dynamic balancing
}

\author{
Roberto Torreggiani ${ }^{1}$, Stefano Paolo Corgnati ${ }^{2}$ \\ ${ }^{I}$ Chief Engineer, Engineering Dept. Giacomini Spa San Maurizio D'Opaglio Novara ITALY \\ ${ }^{2}$ Full Professor of Building Physics Energy Dept.,Politecnico di Torino, Corso Duca degli Abruzzi 24, Torino ITALY
}

\section{Summary}

Having to design and plan building renovations of different nature, from large centralized installations to small apartments, in the context of manifold distribution, especially for radiant applications, the main theme is to balance the flows in a correct and minimally invasive way.

The central point is to intervene effectively without bringing with you the side effects of the unbalancing of flows, that are : irregularity of the power distribution, noise and finally energy unefficiency.

These types of problem are particularly evident in the case of the high differential pressures typical of very large networks.

The operation of the variable flow pump can certainly mitigate this aspect, however by acting only on energy saving, it cannot in fact intervene in the balancing in any way.

The technical approaches are substantially two, the first, more tied to tradition, that is the use of the differential pressure controller in combination with a static balancing valve mounted upstream of a standard manifold, the second more innovative and simple is instead the use of manifolds with integrated cartridge for dynamic balancing in each single circuit.

The application with this type of solution has many operational and managerial advantages.

The dynamic balancing manifolds are equipped with integrated cartridges that regulate and limit the flow to preset values.

The preset flow rate on the single circuit will not exceed, even if there are load changes inside the system due to the closing of other valves or during the first start-up.

Within a minimum and maximum differential pressure range, this operation is completely independent from the differential pressure.

This type of intervention, inserting a dynamic balancing manifold in spite of a standard one, is infinitely simpler than installing differential pressure controllers upstream of the manifolds and ultimately much more precise.

Since the required flow rate can be preset directly on the individual circuit by means of an adjustment key, complicated calculations of pressure drop and balancing are no longer necessary, finally the commissioning time is clearly reduced.

\section{Introduction}

The study as described below has as a target the identification of the potential energy savings due to the correct delivery of thermal loads in the rooms by the use of the manifold system technology with "dynamic balancing of the flow".

The study is expressed through the development of two exemplary cases, indicated as "individual" and "collective". The "individual" case examines the typical situation of the internal 
effects of a single housing unit, a case that can occur with a villa or apartment with an independent system. Conversely, the "collective" case examines the typical situation of collective / multi-family residential buildings (condominiums, for example) with centralized systems where energy saving is linked to the dynamics induced by the energy behavior of the individual units.

\section{Case 1 - Energy savings according to the "individual" model (endogenous)}

Let's consider a housing unit (for example, a single-family home or an apartment with an independent heating system) that uses a low inertia radiant floor as its heating system.

The distribution of the heat-transfer fluid towards the various radiant circuits occurs through a single manifold that represents the starting point of the coils servicing the rooms of two "thermal areas": the living area (kitchen, living room and bathroom) and the sleeping area (bedroom 1, bedroom 2 and family room/den).

Each thermal area is controlled via a zone thermostat located in a room considered the "pilot" room and selected as representative: the living room for the living area and the family room/den for the sleeping area .

The signal received by the thermostats controls the control valves of the corresponding rooms in the living and sleeping areas.

Given that:

$\mathrm{g}$ and $\mathrm{n}$ are the subscripts used to specify respectively the living and sleeping area

*, is the apex used to specify the design conditions

ci, is the fraction of total flow rate of the heat-transfer fluid flowing in the i-th circuit

$\mathrm{p}$, is the subscript used to specify the "pilot" rooms

$\mathrm{np}$, is the subscript used to specify the "non-pilot" rooms

Now, in design condition the overall flow rate is

$$
m * \text { tot }=m * n+m * g
$$

and, by the effect of the size of the rooms and their thermo-physical characteristics, it is distributed in designed conditions according to a specific percentage, for example:

$60 \%$-> living area

$40 \%$-> sleeping area

Therefore:

$$
m * n=x m^{*} \text { tot where } x=0.4 \text {. }
$$

We can now assume that at the time t1, e.g. at 10 a.m., in the pilot room of the living area the Tsp set-point temperature is reached. As a result, the valves in the living area will close thus stopping the flow on the corresponding circuits.

If a "flow rate dynamic balancing" manifold is not used, the flow rate destined to the living area will now be redistributed to the sleeping area (a drop due to an increased loss of pressure can occur, but this effect not considered herein as an initial approximation).

Let us assume that the $\mathrm{m}^{*} \mathrm{n}$ flow rate circulating in the sleeping area circuits is distributed according to a design percentage, for example:

- room 1: $45 \%$ of $m^{*} n$

- room 2: $40 \%$ of $m^{*} n$

-family room/den $15 \%$ of $m * n$ 
The overall flow rate under the new operating conditions circulating in the sleeping area is now:

$m$ 'notte $=m^{*} n+m^{*} g=m *$ tot

where $\mathrm{m}^{*}$ tot is the total design flow rate, which will circulate according to the percentages specified above for each of the three rooms forming the "sleeping" area.

Furthermore, let us consider that by the effect of the intrinsic trend of radiant heating systems to re-balance the power supplied, in the new operating conditions the increase in flow rate corresponds to a reduction in DT' compared to the design DT*:

$$
D T^{\prime}=y D T^{*} \text { where }(1-x)<y<1
$$

Now, let us assume that:

-due to the endogenous and exogenous heat flows, the temperature in the pilot room of the living area remains higher than the set-point temperature for $\mathrm{N}$ hours during the day (for example, from 9:00 a.m. to 4:00 p.m., thus $\mathrm{N}=6$ )

- in the same $\mathrm{N}$ hours, the pilot room of the sleeping area never reached the set-point temperature (consequently, a flow rate exceeding the design one continues to circulate in the "sleeping" circuits)

- whilst in the two other rooms, the temperature exceeds the established temperature for $\mathrm{M}$ hours.

Now, let us express the flow rate in the sleeping area according to the design condition as:

$$
m * \text { notte }=a m^{*} \text { notte }+b m^{*} \text { notte where } a+b=1
$$

where $a$ is the flow rate fraction of the sleeping area circulating in the "pilot" room circuit and $b$ in the "non-pilot" ones.

Similarly: m'notte $=\mathrm{a}$ m'notte $+\mathrm{b}$ m'notte

In the rooms of the "non-pilot" sleeping area (subscript np) therefore, under the design conditions, the thermal power supplied would be equal to:

$$
Q^{*} n p, n=\left(b m^{*} \text { notte }\right) \text { cp } D T^{*}=\left(b \times m^{*} \text { tot }\right) c p D T^{*}
$$

Instead, in the aforementioned condition where the set-point temperature is not reached in the pilot room, the following operating condition would occur:

$Q^{\prime} n p, n=\left(b m^{\prime}\right.$ notte $) c p D T^{\prime}=\left(b m^{*}\right.$ tot $) c p\left(y D T^{*}\right)$, since m'notte $=\mathrm{m}^{*}$ tot

When the desired temperature is reached in the "non-pilot" room, but the thermal power continues to be supplied in the room as the set-point temperature is not reached in the "pilot" room of the sleeping area, we will have the following situation:

1 - a flow rate being supplied to the room exceeding the planned one, expressed as

$$
D m^{\prime} n p, n=b(1-x) m * t o t
$$

2 - a corresponding over-power being supplied to the room equal to:

$$
D Q^{\prime} n p, n=b(1-x) m^{*} \text { tot cp y } D T^{*}
$$

Now assuming that this condition occurs for $M$ hours during the day (where $M<=N$ ) the result in terms of ideal thermal energy $[\mathrm{kWh}]$ during the day under design conditions is:

$$
E^{*} \text { tot }=m * \text { tot } c p D T^{*} 24
$$

while in the presence of over-power supplied for M hours, an overload situation occurs equal to:

$$
D E^{\prime} n p, n=b(1-x) m^{*} \text { tot cp y } D T^{*} M
$$

Consequently, the over-consumption of energy can be expressed as:

$$
\% E^{\prime}=b(1-x) y(M / 24)
$$


By looking at this from the opposite point of view, this over-consumption corresponds to the ENERGY SAVINGS obtainable through the use of a "flow rate dynamic balancing" manifold: ENERGY SAVING \% $\left(\% E^{\prime}\right)=b(1-x)$ y $(M / 24)$

The following table shows, according to a "sensitivity analysis" logic, the results of the potential energy savings obtainable by effect of a change in the parameters affecting the model.

Table - Energy-saving potential associated to a parameter change in the model.

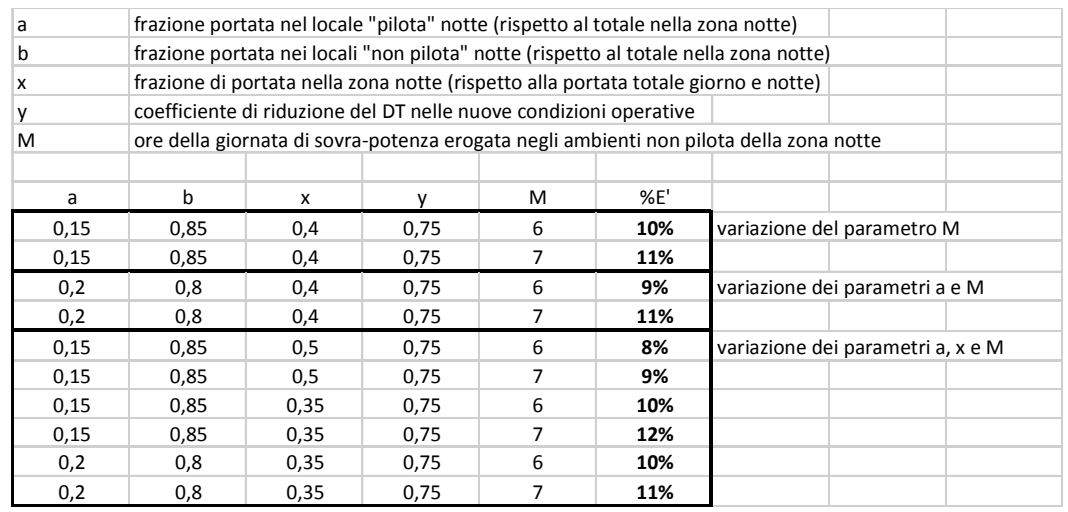

\section{Case 2 - Energy savings according to a "collective" model (ex- ogenous)}

Let us consider a multi-family building split into $\mathrm{z}$ housing units (for example, a condo with 8 apartments; a mezzanine floor plus 3 other floors with 2 apartments per floor), with a centralized heating system serving all housing units, which have a low inertia radiant floor as HVAC system.

The distribution of the heat-transfer fluid from the boiler room to the housing units occurs via a primary circuit of heating hot water that serves the apartment manifolds: each apartment is served by a dedicated manifold.

In each apartment, each manifold is connected to the various radiant circuits whose coils serve the rooms of the apartment (for example kitchen/living room, family room, bathroom, bedroom 1 , and bedroom 2).

Given that: 
$\mathrm{g}$ and $\mathrm{n}$ are the subscripts used to specify respectively the living and sleeping area

$*$, is the apex used to specify the operating conditions of the project

*, the apex used to specify the operating conditions under review

$\mathrm{ci}$, is the total flow rate fraction of the heat-transfer fluid flowing in the $\mathrm{i}$-th circuit

$\mathrm{p}$, is the subscript used to specify the "pilot" rooms

$\mathrm{np}$, is the subscript used to specify the "non-pilot" rooms

$\mathrm{cp}$, is the subscript used to specify the "primary circuit".

$1, \ldots, \mathrm{n}$, is the subscript used to specify the $\mathrm{z}$ housing units (apartments) of the building

I , the subscript used to specify the generic i-th housing unit

Now, let us consider the heating circuit. The total flow rate supplied by the primary circuit according to the design conditions is

$\mathrm{m} *$ tot $=\mathrm{m}^{*} 1+\ldots+\mathrm{m}^{*} \mathrm{n}$

which, by effect of the size of the apartments and their thermo-physical characteristics is dis-

tributed under the planned conditions according to a specific apportionment, for example:

$$
m * i=z i m * t o t
$$

where zi is the total flow rate fraction to the manifold of the $\mathrm{i}$ housing unit, given that:

$$
z 1+\ldots+z n=1
$$

If under design conditions the flow rate is equally distributed over each apartment, then:

$$
z i=z 1=\ldots=z n=1 / n
$$

The thermal power supplied by the system under design conditions shall then be expressed as:

$$
Q^{*} \text { tot }=m^{*} \text { tot cp } D T^{*}=z 1 m^{*} \text { tot cp } D T^{*}+\ldots .+z n m^{*} \text { tot cp } D T^{*}
$$

where DT* is the design temperature gradient on the system terminal.

Now, let us suppose that the $\mathrm{z}$ apartments (e.g. $\mathrm{z}$ equal to 8) are occupied by households having the following profile by way of example:

- Type A apartments (e.g. 3): occupied by retirees who keep the heating system continuously on throughout the day.

- type B apartments (e.g. 5): occupied by families with working parents and school-age children where these families turn off the heating system for a time interval of $\mathrm{H}$ hours, e.g. from 8:00 am to 2:00 pm hours (6 hours off)

If "flow rate dynamic balancing" manifolds are not used, during the $\mathrm{H}$ hours of the heating system turned off by type B apartments, the flow rate previously destined to the various housing units will be redirected to type A housing units (unless there is a drop due to an increased loss of pressure, an effect not considered herein as an initial approximation).

Thus:

where $\mathrm{m}^{*}$ tot is the design total flow rate.

$$
m \text { 'tot, tipo } A=m^{*} \text { tot }
$$

By way of example, let us consider the case of 8 total apartments of which 3 of type A, the latter will have now a total flow rate split into percentages for each of the aforementioned 3 apartments (identified with subscripts $1,3,5$ ). More specifically, it is assumed that:

$$
\begin{gathered}
z i^{*}=z 1=\ldots=z 8=\cos t=1 / 8 \\
z^{\prime} i, \text { tipo } A=z^{\prime} 1=z^{\prime} 3=z^{\prime} 5=\cos t=1 / 3
\end{gathered}
$$

In this case, the flow rate on each of the 3 type $A$ apartments will be equal to:

$$
m^{\prime} 1=m^{\prime} 3=m^{\prime} 5=z^{\prime} i \text {, tipoA } m^{*} \text { tot }=1 / 3 m^{*} \text { tot }
$$

Now let us focus on one of the type A apartments. We can assume that the type A apartment under review is designed as a single "thermal zone", controlled by a single room thermostat placed in a "pilot" room chosen as representative: for example "bedroom 1" with northern exposure, generally characterized by limited exogenous and endogenous effects. 
Under design conditions, let us assume that the flow rate circulating in the circuits of the apartment (reminder: $\mathrm{m} * \mathrm{i}=\mathrm{zi} \mathrm{m} * \mathrm{tot}$ ) is distributed according to a percentage calculated according to the project as follows:

- "pilot" room: $\mathrm{a}=0.2$, therefore $20 \%$ of the flow rate circulating in the apartment.

- other "non-pilot" rooms: $\mathrm{b}=0.8$, thus $80 \%$ of the flow rate circulating in the apartment.

Under the new operating conditions, the total flow rate circulating in the type A apartment is, as shown above:

$$
m^{\prime}=z^{\prime} i \text {, tipoA } m^{*}
$$

where $\mathrm{m}^{*}$ tot is the planned total flow rate that will circulate distributed in percentage ( $\mathrm{z}$ ' $\mathrm{i}$, tipoA ) for each of the 3 type A apartments.

Furthermore, let us consider that by the effect of the intrinsic trend of the radiant heating systems to re-balance the power being supplied, under the new operating conditions the increase in flow rate corresponds to a reduction in DT' compared to the design $\mathrm{DT}^{*}$ :

Now, let us assume that:

$$
D T^{\prime}=y D T^{*} \text { where }(1-x)<y<1
$$

- during the $\mathrm{H}$ hours of turn off time in type B apartments, in the pilot room of the type A apartment the set-point temperature is never reached (consequently during said $\mathrm{H}$ hours a flow rate equal to $\mathrm{m}^{\prime}=\mathrm{z}$ 'i, tipoA $\mathrm{m}^{*}$ higher than the planned one will continue to circulate in the circuits of the type A apartment )

- at the same time in the other "non-pilot" rooms of the type A apartment the temperature exceeds for $\mathrm{M}$ hours (with $\mathrm{M}<=\mathrm{H}$ ) the established temperature desired.

Now, let us express the flow rate of the type A apartment according to the planned condition as:

$$
m^{*}=a m^{*}+b m^{*} \text { being } a+b=1
$$

where, as already defined before, $a$ is the flow rate fraction circulating in the "pilot" room circuit and $b$ in the "non-pilot" ones.

Similarly, under the new operating conditions:

$$
m^{\prime}=a m^{\prime}+b m^{\prime}
$$

Therefore, under the designed operating conditions, the thermal power supplied in the "nonpilot" rooms (subscript np) would be equal to:

$$
Q^{*} n p=\left(b m^{*}\right) c p D T^{*}=\left(b z i * m^{*} t o t\right) c p D T^{*}
$$

Instead, under the operating conditions described above, where the set-point temperature is never reached in the pilot room during the $\mathrm{H}$ hours when the type B apartments are switched off, the following operating condition would occur:

$$
Q^{\prime} n p=\left(b m^{\prime}\right) c p D T^{\prime}=\left(b z^{\prime} i \text {, tipoA } m^{*} \text { tot }\right) c p\left(y D T^{*}\right)
$$

With the new operating conditions, the following condition occurs in the "non-pilot" rooms: 1 - the flow rate supplied to the room exceeds the planned one, expressed as

$$
D m^{\prime} n p=b\left(z^{\prime} i \text {, tipo } A-z i^{*}\right) m^{*} \text { tot }
$$

2 - with a corresponding over-power supplied to the apartment equal to:

$$
D Q{ }^{\prime} n p, n=b\left(z^{\prime} i \text {, tipo } A-z i^{*}\right) m^{*} \text { tot cp y } D T^{*}
$$

In terms of ideal thermal energy [kWh] supplied during the day under the planned conditions, the result is:

$$
E^{*} t o t=(b z i * m * t o t) c p D T^{*} 24
$$


while with an over-power supplied for $M$ hours (where $M<=H$ ), we will experience an overload equal to:

$$
D E^{\prime} n p, n=b\left(z^{\prime} i \text {, tipo } A-z i^{*}\right) m^{*} \text { tot cp y } D T^{*} M
$$

Consequently, over-consumption in the type A apartment under review can be expressed as:

$$
\% E^{\prime}=\left[\left(z^{\prime} i, \text { tipo } A-z i^{*}\right) / z i^{*}\right] \text { y }(M / 24)
$$

By looking at this from the opposite point of view, this over-consumption corresponds to the ENERGY SAVINGS obtainable in the type A apartment by using a "flow rate dynamic balancing" manifold:

$$
\text { ENERGY SAVING \% (\% E') tipo } A=\left[\left(z^{\prime} i, \text { tipo } A-z i^{*}\right) / z i^{*}\right] \text { y }(M / 24)
$$

Considering that each type A apartment has the same thermal dynamics during the day, when "flow rate dynamic balancing" manifolds are installed, during the day all the type A apartments will contribute to reduce the energy consumption of the centralized system, amounting to:

$$
D E^{\prime} \text { tot }=\Sigma D E^{\prime} j \text { app } \text {, tipo } A j=1
$$

with corresponding energy savings on the centralized system (thus for the entire condo) in the amount of:

$$
\text { ENERGY SAVING \% (\% E')tot = D E'tot / E*tot }
$$

The following table shows, according to a "sensitivity analysis" logic, the results of the total energy saving potential on the entire condo (\% E')tot linked to the change of parameters affecting the model with reference to the following two combinations of occupant profiles1:

\section{CASE 1}

Total number of apartments $=8$

Number of apartments occupied by retirees ( Type A apartment $)=2$

Number of apartments occupied by singles and/or families without children $(H=7 ; M=6)=4$

Number of apartments occupied by families with children $(\mathrm{H}=5 ; \mathrm{M}=4)=2$

\section{CASE 2}

Total number of apartments $=8$

Number of apartments occupied by retirees ( Type A apartment, $\mathrm{H}=0$ ) $=3$

\begin{tabular}{|c|c|c|c|c|c|c|c|c|}
\hline & Pensionati & Single/Fam. senza figli & Famiglia con figli & & & & & \\
\hline $\mathrm{n}^{\circ}$ Appart. & $\mathrm{n}^{\circ}$ Appart. & $n^{\circ}$ Appart. / M & $n^{\circ}$ Appart. / M & $\mathrm{zi}^{*}$ & $z^{\prime} i$, tipoA & b & $y$ & $\begin{array}{l}\text { Risparmio } \\
\text { energetico di } \\
\text { Condominio }\end{array}$ \\
\hline 8 & 3 & $3 / 6$ & $2 / 4$ & 0,125 & 0,333 & 0,8 & 0,7 & $14 \%$ \\
\hline 8 & 2 & $4 / 6$ & $2 / 4$ & 0,125 & 0,5 & 0,8 & 0,63 & $25 \%$ \\
\hline
\end{tabular}

Number of apartments occupied by singles and/or families without children $(\mathrm{H}=7 ; \mathrm{M}=6)=3$

Number of apartments occupied by families with children $(\mathrm{H}=5 ; \mathrm{M}=4)=2$

Table - Total energy-saving potential, referred to the entire condo, associated to parameter changes in the model.

\begin{tabular}{|l|l|l|}
\hline zi* & frazione della portata totale del sistema nell'appartamento tipo A in studio (in condizioni di progetto) \\
\hline z'i,tipoA $^{*}$ & frazione della portata totale del sistema nell'app. tipo A in studio nelle nuove condizioni operative \\
\hline b & frazione portata nei locali "non pilota" del locale tipo A (rispetto al totale circolante nel locale tipo A) \\
\hline y & coefficiente di riduzione del DT nelle nuove condizioni operative \\
\hline M & ore della giornata di sovra-potenza erogata negli ambienti non pilota della zona notte \\
\hline
\end{tabular}


The profiling adopted is deduced from the data presented by the ISTAT statistics, a distribution of households can be found attributable to the following percentage profiling: pensioners $30 \%$, single / couple without children $40 \%$, couple with children $30 \%$.

\section{Summary of Results}

The model developed allowed estimating the energy-saving potential associated with the use of a "flow rate dynamic balancing" manifold .

With reference to the two cases reviewed and background conditions (input and characteristic parameters of the model) reviewed:

- the "individual" case, providing an example of energy savings for housing units with an independent heating system, showed energy savings up to $12 \%$;

- the "collective" case, providing an example of energy savings for multi-family buildings with a centralized heating system, showed energy savings up to $25 \%$.

\section{Conclusions}

The frontier of development, with a view to enhancing energy savings, sees dynamic flow balancing systems to have primary place and position.

But they do not proceed alone but certainly coupled with low thickness and low inertia systems.

It is in fact through the combination of the effect of low thermal inertia and the dynamic control of the flow rate that the best effects are obtained.

The dynamic balancing of the flow certainly has a potential energy impact that is important as demonstrated in the study we introduced above.

The target is to make the balance usable and easy to apply in new and existing applications. A system with dynamic balancing manifolds is able to keep the flow rate always balanced for all system circuits, thus contributing to the reduction of overflows and, consequently, to an appreciable energy saving.

Overall dimensions and applications are those of a standard manifold but the cartridge installed in each circuit operates the following functions:

- flow control: when the pressure varies, due to the opening or closing of some circuits, the diaphragm of the cartridge valve intervenes by varying its passage section and adapting the flow to the preset value, even in the presence of high differential pressures.

- flow rate pre-setting: it is possible to set the maximum design flow rate for each individual circuit, which must be kept constant;

- room temperature optimization: the combination with electrothermic heads and room thermostats allows to optimize temperature management in the various rooms.

It is indeed through simple and reliable applications such as manifolds that dynamic balancing technology can make us achieve a step forward in cost optimality approach. 


\section{References}

1 . Torreggiani Roberto "Collettori Serie DB con Bilanciamento Dinamico della Portata" Ingenioweb (2021)

2. Torreggiani Roberto "Bilanciamento Dinamico della Portata e sistemi radianti a basso spessore" Ingenioweb (2020)

3. Stefano Paolo Corgnati "Potenzialita' di risparmio energetico legate all'utilizzo del collettore con Bilanciamento Dinamico della Portata" (2020) 